\title{
Design of Vision Guided Intelligent Handing System for Inner Ring and Outer Ring of Railway-bearing
}

\author{
Dapeng Meng ${ }^{1, \mathrm{a}}$, Jinsong $\mathrm{Du}^{1}, \mathrm{Xu} \mathrm{Yang}^{1}$, Xinye Wang ${ }^{1}$ and Fei Liu ${ }^{1}$ \\ ${ }^{1}$ Shenyang Institute of Automation, Chinese Academy of Sciences, Feiyun Road No19, Shenyang, China
}

\begin{abstract}
More and more special automation equipment had been developed with progress of technology. All kinds of nonstandard equipment were applied to the specific occasion. A vision guided intelligent handing system in the paper was specially designed for carrying the inner ring and outer ring of railwaybearing from material handcart to the testing line. Robot, visual positioning, pneumatic clamping jaw, servo motor and PLC were integrated into this handing system. The visual system was led to take pictures for the inner and outer rings by robot, and transfer the location data of rings to the PLC, the brain of the whole system. The PLC controlled robot and pneumatic clamping jaw finish the handing process through accurate calculation.
\end{abstract}

\section{Introduction}

Nonstandard equipment was one kind of privately made, and especial. They were designed and manufactured according to the needs of customers, and used on special occasion. With the increasing integration of automation, nonstandard equipment had drawn more and more attention $[1,2,3]$.

A kind of nonstandard equipment was introduced in the paper, specially designed for carrying rings of railway bearing from handcart to the testing line accurately. The equipment designed in the paper could take the place of manual handing relying on highly integrated automation design.

\section{Mechanical Structure}

The Figure 1 was the 3D model diagram of the mechanical structure. The handing system was mainly made up of electronic control system, robot, vision system, inner ring pneumatic jaw, outer ring pneumatic jaw, servo motor, etc.

Electronic control system (No.1) including PLC and other electrical components was the core of the whole handing system, just like the brain of human beings, collecting information and issuing instructions at the same time. Vision system (No.2) was made up of light source and camera was fixed on an iron shelf, like one eye of human, transferring the position data of the ring it shot to the PLC. Robot (No.3) who had 6 degree of freedom was the carrier for carrying, like the arm of human. Two jaws (No.4) fixed on the flange of the robot were used to pull up inner rings (No.6) and outer rings (No.7) from inside, just like hands of human. Servo motors (No.5) would drive the pneumatic jaws to grab the partition between each layer when finished handing the last ring of each layer. And the partition would be put into the recycling trolley automatically.

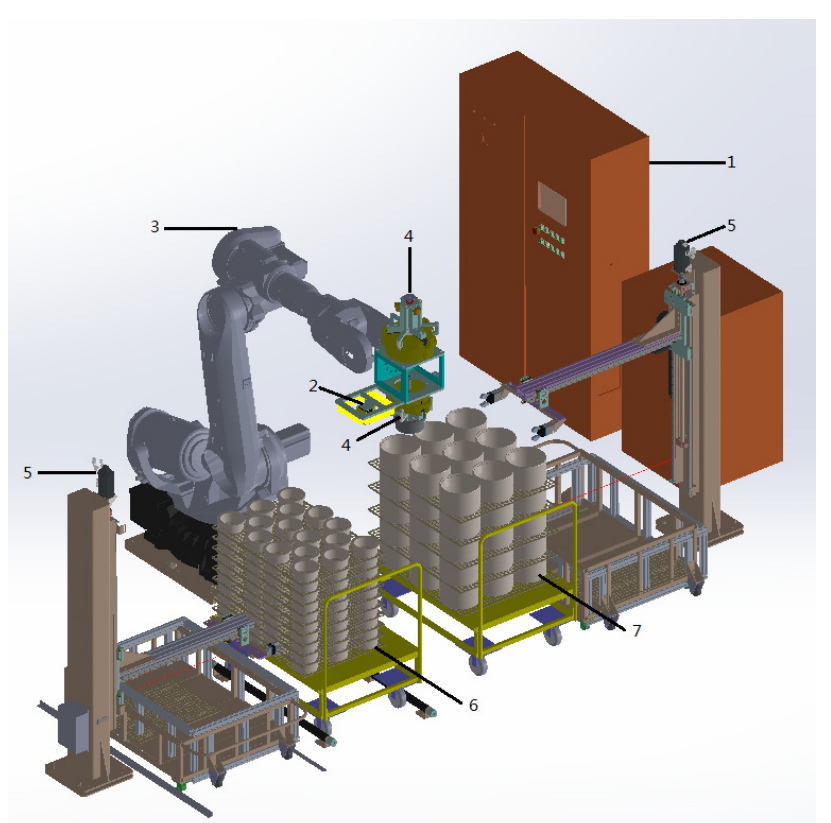

Figure 1. 3D Model Diagram of the Mechanical Structure.

The material of inner and outer rings was bearing steel G20CrMo, which was very tough and undeformable. But in order to ensure the accuracy of the test results, some nylon blocks were mounted on the surface of the jaws, which could prevent the surface of rings scratched. The handcarts would be restricted to a certain area, which could prevent the rings from going beyond the view of camera.

\section{Electrical Structure and Software}

\footnotetext{
* Corresponding author: ${ }^{\mathrm{a}}$ mengdapeng@sia.cn
} 
Electrical structure was the core part of the whole handing system. Programmable Logic Controller (PLC) as the commander of the electrical structure was a kind of digital computing device designed for industrial production. It was widely used in industry because of high reliability, strong anti-interference ability, strong applicability, high performance price ratio and convenient maintenance [4].

\subsection{Electrical Topology}

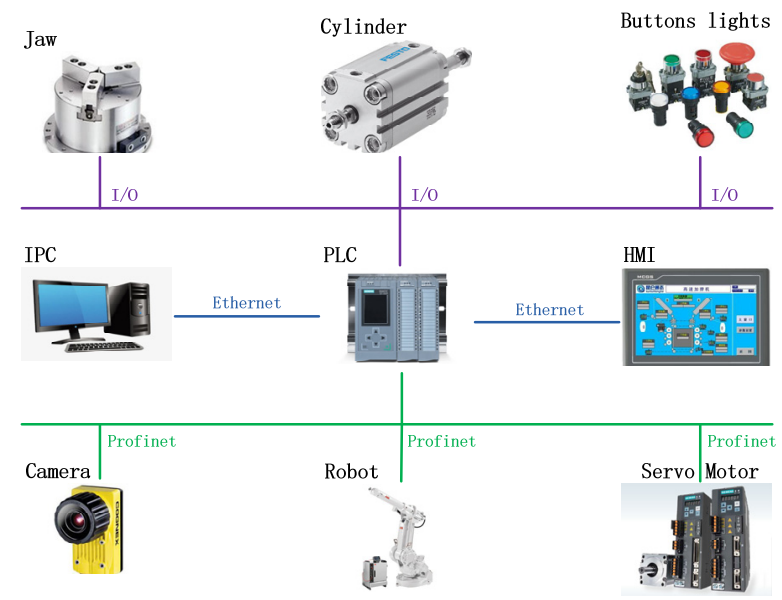

Figure 2. Electrical Topology Diagram.

Figure 2 was the electrical topology diagram of the system. S7-1500 series PLC was applied to control the whole handing system, which was one of the most advanced PLC produced by SIEMENS.

PLC, camera, robot and servo motors were configured in the same network together through the PROFINET protocol, which was a network configuration mode without lots of cable. They transmitted data and instructions through the network, which was not only fast, but did not occupy I/O module of PLC.

PLC could communicate with jaws, cylinders and other low voltage electrical components such as magnetic switches, solenoid value, buttons and indicator lights by I/O cable.

At the same time, the status of the handing system would be displayed on the HMI, which could be edited many interfaces. And the handing system could be operated manually through HMI when running in the manual state. All parameters PLC edited could also be modified through HMI.

PLC communicated with industrial personal computer (IPC) through Ethernet constantly, who was the host computer. IPC would collect information from PLC and transfer instructions to PLC at the same time, just like the commander in chief.

Table 1 was the main hardware configuration details of the handing system.
Table 1. Main hardware configuration details.

\begin{tabular}{|c|c|c|}
\hline Name & Model & Brand \\
\hline PLC & S7-1500 series & SIEMENS \\
\hline Servo Motor & V90 series & SIEMENS \\
\hline Robot & 1600 series & ABB \\
\hline Vision System & IS7000 series & COGNEX \\
\hline HMI & TCP1061Ti & MCGS \\
\hline Cylinder & & FESTO \\
\hline Jaw & - & SCHUNK \\
\hline
\end{tabular}

\subsection{Configuration and Procedure}

Figure 3 was the configuration of the hardware, edited by software TIA Portal V14. PLC who was the master station connected with two drivers of V90 servo motors, vision system and communication card of robot through PROFINET, after their IP addresses were set up in the same segment. The coordinate system of the camera and the robot must be unified through the software of vision system.

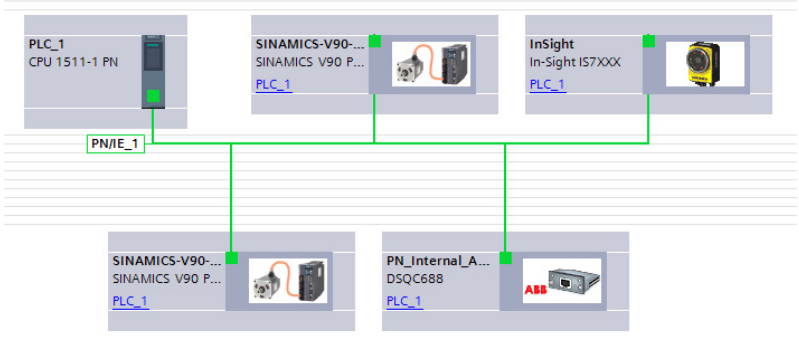

Figure 3. Configuration of the Hardware.

Highly integrated automation made human work easier. Before the movement of handing system, the instruction obtained from the testing line must be sent to the PLC by the IPC. PLC would drive robot move to the position of taking photo for first inner ring, if the instruction was inner ring.

Camera would take a picture for the inner ring, and send the position coordinates of $\mathrm{X}$ and $\mathrm{Y}$ axes to PLC. The coordinate data had to be as a result of distortion correction, ensure the minimum error of data.

Then the robot would get the coordinates data of the inner jaw from PLC. The robot with the jaw would move to the specified position exactly, where was above the circle center of the inner ring [5].

The robot would send the ring to the specified location on the inner testing line, after the inner ring was clamped by the inner jaw.

If the ring was the last one of this layer, the servo motor would drive the double claw to remove the partition between the layers. And if the ring was the last 
one of this handcart, PLC would tell the IPC this handcart had been empty.

The process of the inner ring and the outer ring was nearly the same. There was a little difference the No.6 axis of robot would rotate $180^{\circ}$ after taken picture. The inner ring and outer ring must be clamped by different jaw because of their different size.

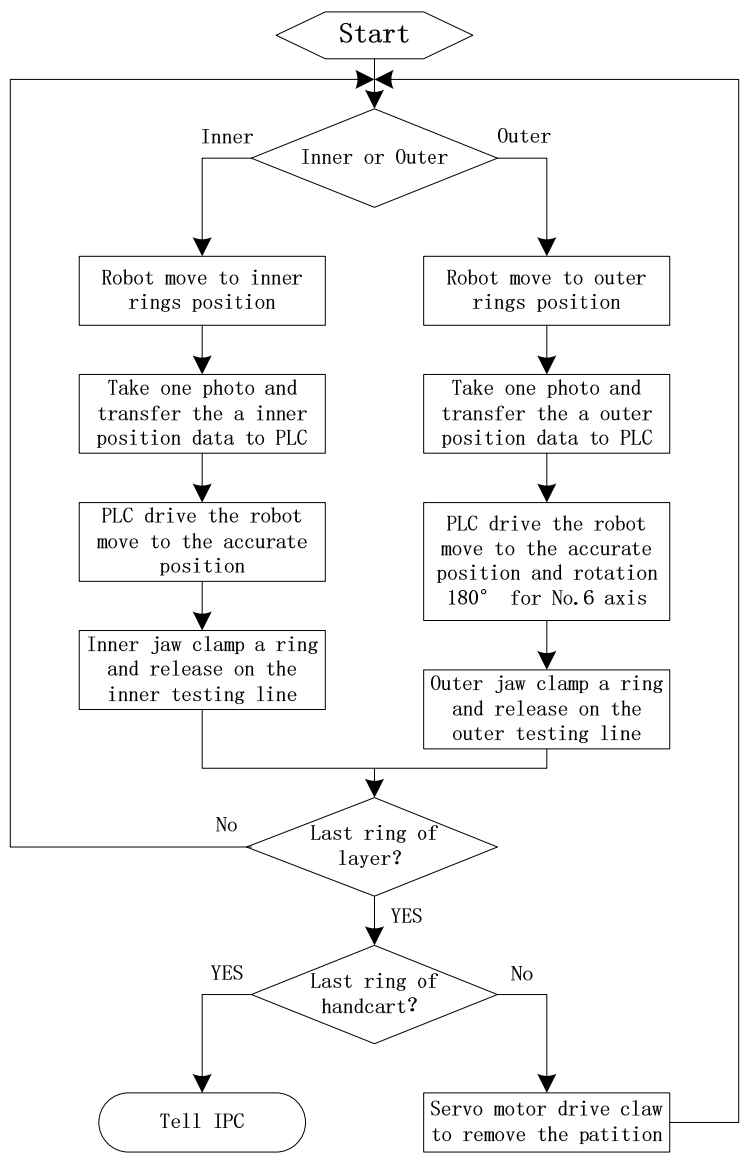

Figure 4. Brief Flow Chart.

Figure 4 was the brief flow chart of the handing system.

The handing system was high-speed, high-efficiency, high-flexibility. It took only 14 seconds to move one inner ring from the handcart to the testing line, as well as 17 seconds for one outer ring. And it could be adapted to multiple types of inner rings and outer rings of railwaybearing.

Table 2 was the list of debugging software used in the handing system.

The configuration of PLC, camera, robot and servo motors was done by the software TIA Portal V14 sp1, as well as the main logic program.

The coordinate transformation between the robot and the vision system was edited in the software In-Sight Explorer 5.5, which would also collect images and compute the coordinates of the ring center [6].

The moving path of the robot was edited by software RobotStudio 5.61. And the parameters of tool and load could be set by FlexPendant of robot.

The configuration of the servo controllers was accomplished by the software V-ASSISTANT-V1-05-00, including the IP, the electrical and mechanical parameters of the servo motor.
All parts of interaction between the workers and the handing system were edited by the software McgsSetE 7.7. The state and the operation mode of the handing system would be displayed on the interface of HMI.

Table 2. Software list.

\begin{tabular}{|c|c|}
\hline Hardware & Software version \\
\hline PLC & TIA Portal V14 sp1 \\
\hline Camera & In-Sight Explorer 5.5 \\
\hline Robot & RobotStudio 5.61 \\
\hline Servo & V-ASSISTANT-V1-05-00 \\
\hline HMI & McgsSetE 7.7 \\
\hline
\end{tabular}

\section{Summary}

The paper had designed a vision guided intelligent handing system, which was exclusively used for carrying inner and outer rings of railway-bearing. It could automatically finish the work of carrying the whole handcart, including removing partitions between layers automatically.

The handing system had been integrated with PLC, robot, vision system, servo and other high-end equipment, which made the work simple and efficient. They were configured by PROFINET bus in one network. Complex movement function was realized by succinct hardware connection.

It could not only save manpower costs, but also improve efficiency. It could be part of the intelligent factory, as well as fit the plan of China Made 2025.

Figure 5 was picture of the hardware. The handing system designed in the paper had already been used in a large domestic bearing manufacturer.

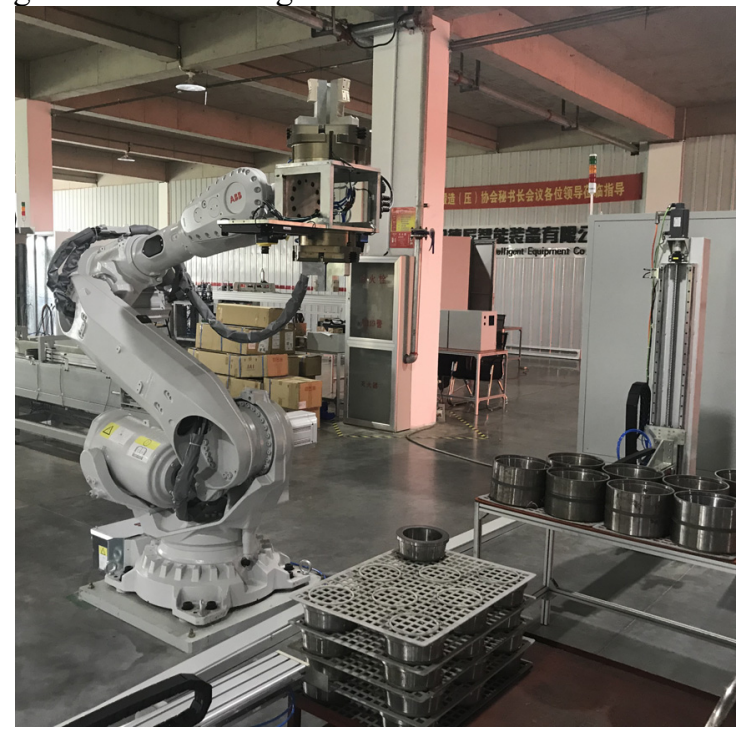

Figure 5. Picture of Hardware. 


\section{Acknowledgements}

This work was supported by the Project of Intelligent Manufacturing Integrated Standardization and New Model Application (A New Intelligent Manufacturing Model for the New Generation of Rail Bearing), belonging to Ministry of Industry and Information Technology of the People's Republic of China.

\section{References}

1. Yao Cheng,Ning Zhou,Weihua Zhang,Zhiwei Wang. Application of an improved minimum entropy deconvolution method for railway rolling element bearing fault diagnosis[J]. Journal of Sound and Vibration,425(2018)

2. Yifan Li,Xihui Liang,Jianhui Lin,Yuejian Chen,Jianxin Liu. Train axle bearing fault detection using a feature selection scheme based multi-scale morphological filter[J]. Mechanical Systems and Signal Processing,101(2018)

3. Ping Wang,Li Wang,Rong Chen,Jinhui Xu,Jinmang $\mathrm{Xu}$,Mingyuan Gao.Overview and outlook on railway track stiffness measurement[J].Journal of Modern Transportation, 89-102(2016,24(02))

4. Da-peng MENG. A New Tightening Device Used for Automobile Differential Mechanism[A]. Science and Engineering Research Center.Proceedings of 2017 2nd International Conference on Applied Mechanics, Electronics and Mechatronics Engineering(AMEME 2017)[C].Science and Engineering Research Center,4(2017)

5. CHEN Yuzhen,XIE Fugui,LIU Xinjun,ZHOU Yanhua.Error Modeling and Sensitivity Analysis of a Parallel Robot with SCARA(Selective Compliance Assembly Robot Arm) Motions[J].Chinese Journal of Mechanical Engineering, 693-702(2014,27(04))

6. Saeed Toosizadeh. A Novel Chaotic Vision Modeling for Mobile Robots based on Two Dimensional Chaos Optimization[A]. International Association of Computer Science and Information Technology (IACSIT),5(2011) 